\title{
A NONNEGATIVE MATRIX FACTORIZATION SCHEME FOR DIGITAL IMAGE WATERMARKING
}

\author{
Mohammadreza Ghaderpanah and A. Ben Hamza \\ Concordia Institute for Information Systems Engineering \\ Concordia University, Montréal, Quebec, H3G 1T7, Canada
}

\begin{abstract}
We present a new scheme for digital watermarking and secure copyright protection of digital images using nonnegative matrix factorization and singular value decomposition approaches. The proposed method improves the performance of the data embedding system effectively, and it is resistant to a variety of intentional attacks and normal visual processes. The experimental results clearly illustrate the much improved performance of the proposed watermarking methodology in comparison with existing techniques.
\end{abstract}

\section{INTRODUCTION}

The recent explosive growth of digital multimedia data and the development of electronic commerce and online services has triggered the need for the protection of multimedia contents. To tackle this problem, watermark embedding in digital multimedia could help in providing proof of origin and distribution of content. Watermarking refers to the process of adding a structure called a watermark to the original data object. Such a watermark can be used for several purposes including copyright protection, fingerprinting, broadcast monitoring, and data authentication.

A basic image watermarking algorithm consists of a cover image, a watermark structure, an embedding algorithm, and an extraction algorithm. A variety of watermarking techniques have been proposed for multimedia protection, and in particular for digital images [46]. These techniques can be divided into two main categories according to the embedding domain of the cover image: spatial domain methods and transform domain methods [6]. The spatial domain methods are the earliest and simplest watermarking techniques but have a low information hiding capacity, and also the watermark can be easily erased by lossy image compression. On the other hand, the transform domain approaches insert the watermark into the transform coefficients of the image cover, yielding more information embedding and more robustness against watermarking attacks. Recently, an SVD-based watermarking technique and its variants have been proposed [7-9]. The main idea of these approaches is to find the SVD of a cover image or the SVD of each block of the cover image, and then modify the singular values to embed the watermark. In [7], a hybrid non-blind watermarking scheme based on the DWT, the DCT and the SVD was proposed. This method consists of decomposing the cover image into four transformed sub-bands, then the SVD is applied to each sub-band, followed by modifying the singular values of the transformed sub-bands with the singular values of the visual watermark. This modification in all frequencies provides more robustness against different attacks [7]. Another SVD-block based watermarking scheme was proposed in [9], and it embeds the watermark in two layers. In the first layer, the cover image is divided into small blocks and the singular values of the watermark are embedded in those blocks. In the second layer, the cover image is used as a single block to embed the whole watermark. One major weakness of SVD is that it produces low rank bases which do not respect the nonnegativity of the cover image. Nonnegative matrix factorization (NMF) was introduced in $[1,2]$ to overcome this limitation without significantly increasing the error of the associated approximation, and it has been shown to be an effective tool in many areas including spectroscopy [3].

In this paper we introduce a new method for digital watermarking and secure copyright protection of digital images. The proposed watermarking scheme is based on NMF and SVD approaches, and it improves the performance of the data embedding system effectively. It is also resistant to a variety of intentional attacks and normal visual processes.

The rest of this paper is organized as follows. In the next section, we introduce the proposed scheme and we describe in more details the main steps of the watermark embedding and extraction algorithms. In Section 3, we present some experimental results to demonstrate the much improved performance of the proposed method in comparison with existing techniques, and 
also to show its robustness against the most common attacks. Finally, we conclude in Section 4.

\section{PROPOSED METHOD}

\subsection{Nonnegative Matrix Factorization (NMF)}

One major drawback of SVD is that the basis vectors may have both positive and negative components, and the data are represented as linear combinations of these vectors with positive and negative coefficients. In many applications, the negative components contradict physical realities. To address this problem, the NMF approach was proposed to search for a representative basis with only nonnegative vectors $[1,2]$. The NMF approach can be formulated as follows. Given a cover image $C$ of size $m \times m$, we can approximately factorize $C$ into the product of two nonnegative matrices $B$ and $H$ with sizes $m \times r$ and $r \times m$ respectively, that is $C=B H$, where $r \leq m$. The nonnegative matrix $B$ contains the NMF basis vectors, and the nonnegative weight matrix $H$ contains the associated coefficients (nonnegative weights). To measure the quality of the approximation factorization $C=B H$, a cost function between $C$ and $B H$ needs to be optimized subject to non-negativity constraints on $B$ and $H$. This is done by minimizing the $\mathcal{I}$-information divergence given by

$$
\mathcal{I}(C \| B H)=\sum_{i j}\left(C_{i j} \log \frac{C_{i j}}{(B H)_{i j}}-C_{i j}+(B H)_{i j}\right),
$$

which yields the following multiplicative update rules

$$
\begin{aligned}
& H_{k j} \leftarrow H_{k j} \frac{\sum_{i} B_{i k} C_{i j} /(B H)_{i j}}{\sum_{i} B_{i k}}, \\
& B_{i k} \leftarrow B_{i k} \frac{\sum_{j} H_{k j} C_{i j} /(B H)_{i j}}{\sum_{j} H_{k j}},
\end{aligned}
$$

where the matrices $B$ and $H$ are initialized as nonnegative random matrices, and the updates are done alternatively, that is after updating one row of $H$, we need to update the corresponding column of $B$.

\subsection{Proposed NMF Watermarking Scheme}

The proposed watermarking scheme is based on NMF and SVD factorization approaches. The key idea of this proposed scheme is to apply NMF to the watermark image, and also to each block of the cover image. The embedding and extraction algorithms are illustrated in the block diagrams of Fig. 1 and Fig. 2.

\section{Watermark embedding algorithm:}

1) Divide the cover image into blocks of size $\ell \times \ell$.
2) To each block $L$, apply NMF: $L=B_{\ell} H_{\ell}$, followed by an SVD to the weight matrix: $H_{\ell}=U_{\ell} \Sigma_{\ell} V_{\ell}^{\prime}$.

3) Apply NMF to the watermark: $W=B_{w} H_{w}$, followed by an SVD to the weight matrix: $H_{w}=$ $U_{w} \Sigma_{w} V_{w}^{\prime}$, where $\Sigma_{w}=\operatorname{diag}\left(\lambda_{w i}\right)$.

4) Modify $\lambda_{\max }$ according to $\lambda_{i}^{d}=\lambda_{\max }+\alpha \lambda_{w i}$, where $\lambda_{\max }$ denotes the largest $\mathrm{SV}$ of $H_{\ell}, \alpha$ is a scaling factor, and $\lambda_{i}^{d}$ denotes the distorted $\mathrm{SV}$ of $H_{\ell}$.

5) Use all the distorted blocks $L^{d}=B_{\ell} H_{\ell}^{d}$, where $H_{\ell}^{d}=U_{\ell} \Sigma_{\ell}^{d} V_{\ell}^{\prime}$ and $\Sigma_{\ell}^{d}=\operatorname{diag}\left(\lambda_{i}^{d}\right)$, to produce the watermarked image.

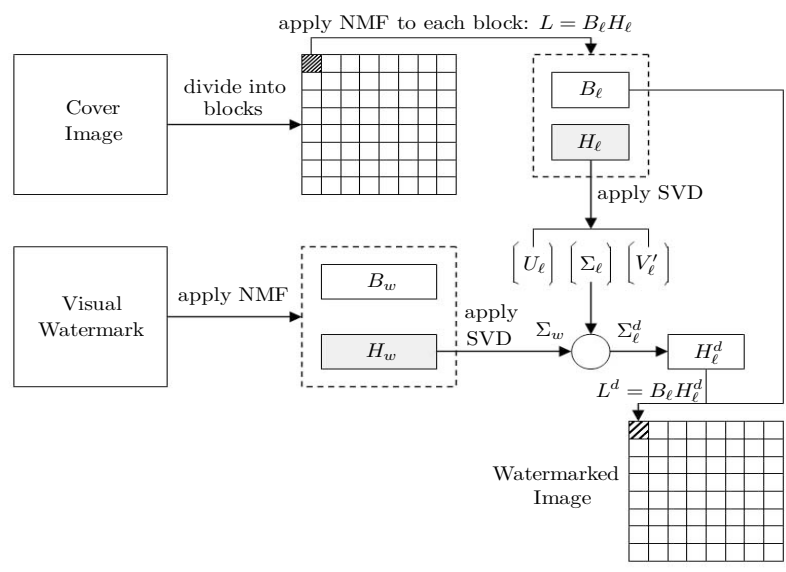

Fig. 1. Watermark embedding algorithm.

\section{Watermark extraction algorithm:}

1) Divide the watermarked image into blocks

2) To each block $K$ of the watermarked image, apply NMF: $K=B_{k} H_{k}$, followed by an SVD to the weight matrix: $H_{k}=U_{k} \Sigma_{k} V_{k}^{\prime}$.

3) Extract the singular values from each block using $\hat{\lambda}_{w i}=\left(\lambda_{i}^{d}-\lambda_{\max }\right) / \alpha$, where $\lambda_{i}^{d}$ are the SVs of $H_{k}$, and $\lambda_{\max }$ is the largest $\mathrm{SV}$ of $H_{\ell}$ which is saved in each block of the secret key (cover image).

4) Construct the watermark image $\widehat{W}=B_{w} \widehat{H}_{w}$, where $\widehat{H}_{w}=U_{w} \widehat{\Sigma}_{w} V_{w}^{\prime}$. Note that $U_{w}$ and $V_{w}$ are saved in the secret key during the embedding stage, and $\widehat{\Sigma}_{w}=\operatorname{diag}\left(\hat{\lambda}_{w i}\right)$ is obtained from step $\left.\mathbf{3}\right)$.

\section{EXPERIMENTAL RESULTS}

We have tested the proposed watermarking scheme and the SVD-based watermarking schemes in a variety of 


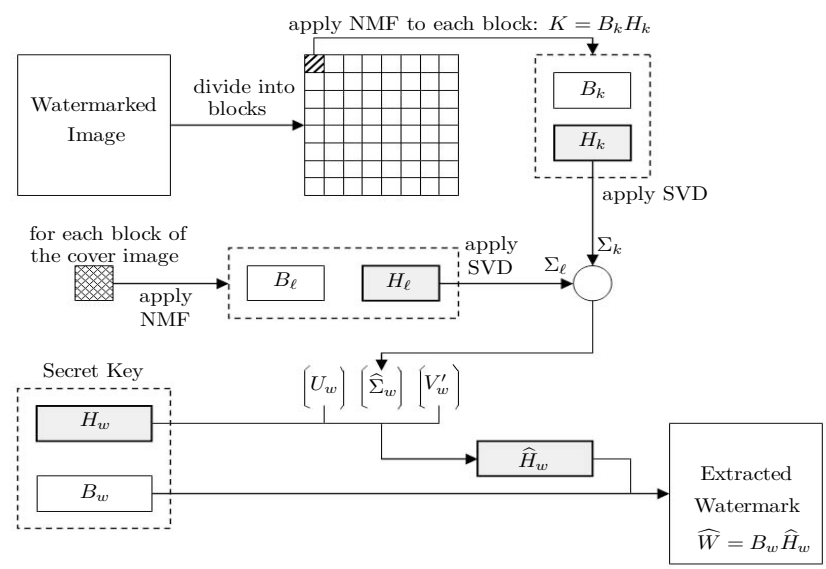

Fig. 2. Watermark extraction algorithm.

experiments under different attacks including JPEG compression, gaussian noise, impulsive noise, multiplicative noise, median filtering, and sharpening. Fig. 3 shows a $256 \times 256$ cover image 'Lena', a $256 \times 256$ visual watermark 'Cameraman', and three extracted watermarks using the SVD scheme, the block-based SVD scheme $(8 \times 8$ block size $)$, and the proposed watermarking method $(8 \times 8$ block size $)$. The scaling factor $\alpha$ was chosen to satisfy two conditions: (i) the invisibility property, and (ii) the highest correlation coefficient between the visual watermark and the extracted watermark before applying any attacks. For the SVD scheme and the proposed method, we set the scaling factor $\alpha$ to 0.08 . For the block-based SVD scheme, we used $c=0.1$, and $\alpha=0.4$. Fig. 3(c)-(e) show the extracted watermarks before applying any attack to the watermarked image. Note that in this experiment the correlation coefficients between the cover image and the watermarked image are all equal to 0.9998, and the correlation coefficients between the visual watermark and the extracted one are all equal to 1.0.

We tested our proposed scheme against a wide range of attacks including JPEG compression, Gaussian noise, impulsive noise, multiplicative noise, median filtering, and sharpening. Fig. 4 presents the watermarked image affected with these attacks. The corresponding extracted watermarks for the proposed scheme, block-based SVD and SVD techniques are shown in Fig. 5. The visual comparison between the extracted watermarks of the three watermarking techniques clearly shows that the proposed scheme gives the best results against all the listed attacks.

The correlation coefficient results are depicted in Fig. 6, where the performance of our proposed approach over the SVD scheme and block-based SVD scheme is clearly demonstrated. We also tested our

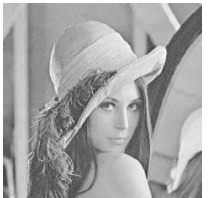

(a)

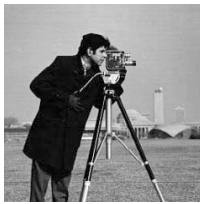

(d)

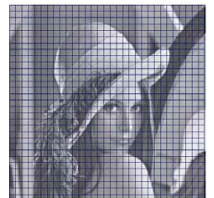

(b)

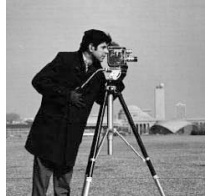

(e)

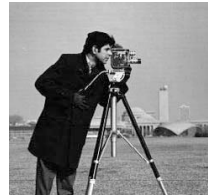

(c)

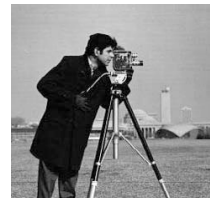

(f)
Fig. 3. First row: (a) Cover image, (b) $8 \times 8$ blocks, (c) visual watermark. Second row: Extracted watermark using (d) SVD-based scheme, (e) block-based SVD scheme, and (f) the proposed method.

proposed algorithm using different block sizes as depicted in Fig. 7. We achieve more robustness against most of the attacks by using a block size $32 \times 32$.

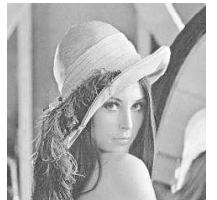

(a)

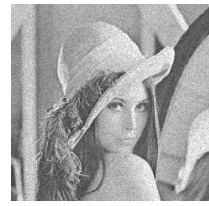

(d)

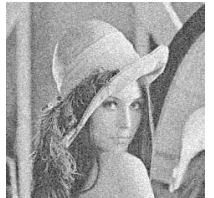

(b)

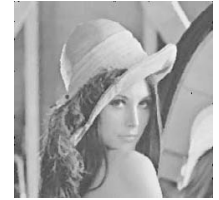

(e)

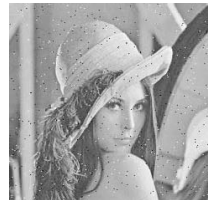

(c)

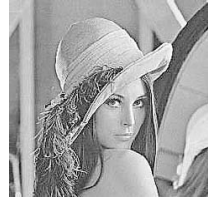

(f)
Fig. 4. Watermarked image: (a) JPEG compression 50:1, (b) Gaussian noise 0.006, (c) impulsive noise 0.01, (d) multiplicative noise 0.01 , (e) median filtering, (f) sharpening 0.5 .

\section{CONCLUSIONS}

We presented a novel hybrid watermarking technique for embedding visual watermarks into digital images using a combination of NMF and SVD approaches. The proposed scheme improves the performance of the data embedding system effectively, and it is resistant to a variety of intentional attacks and normal visual processes. Our experimental evaluations clearly show that the proposed watermarking technique outperforms the current SVD-based watermarking schemes. 
Proposed scheme

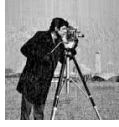

(a) (d)

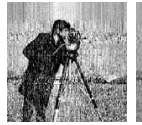

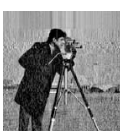

(g)

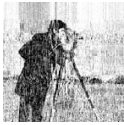

(j) (m)

(p)

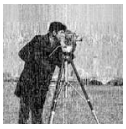

Block-based SVD scheme

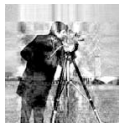

(b)

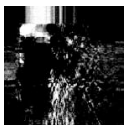

(e)

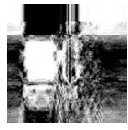

(h)

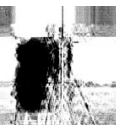

(k)

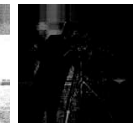

(n)
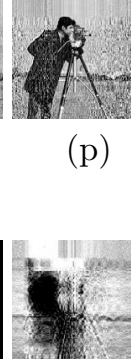

(q)
SVD-based scheme

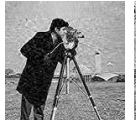

(c)

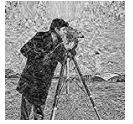

(f)

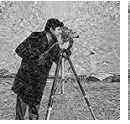

(i)

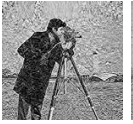

(1)

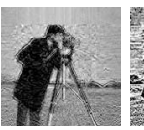

(o)

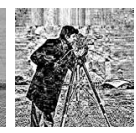

(r)
Fig. 5. Extracted watermark: (a)(b)(c) JPEG compression 50:1, (d)(e)(f) Gaussian noise 0.006, (g)(h)(i) impulsive noise $0.01,(\mathrm{j})(\mathrm{k})(\mathrm{l})$ multiplicative noise 0.01 , $(\mathrm{m})(\mathrm{n})(\mathrm{o})$ median filtering, (p)(q)(r) sharpening 0.5.

\section{Acknowledgments}

This work was supported in part by NSERC Discovery and NATEQ Nouveaux Chercheurs.

\section{REFERENCES}

[1] D. Lee and H. Seung, "Learning the parts of objects by nonnegative matrix factorization," $\mathrm{Na}$ ture, vol. 401, pp. 788-791, 1999.

[2] D. Lee and H. Seung, "Algorithms for nonnegative matrix factorization," Adv. in Neural Info. Proc. Systems, 13, 2000.

[3] A. Ben Hamza and David Brady, "Reconstruction of reflectance spectra using robust nonnegative matrix factorization," IEEE Trans. Signal Processing, to appear 2006.

[4] I. J. Cox, J. Kilian, T. Leighton, and T. Shamoon "Secure spread spectrum satermarking for multimedia," IEEE Trans. Image Processing, vol. 6, no. 12, pp. 1673-1687, 1997.

[5] N. Memon and P. Wong, "Digital watermarks: protecting multimedia content," Communications of the ACM, vol. 47, no. 7, pp. 35-43, 1998.

[6] F. Hartung and M. Kutter, "Multimedia watermarking techniques," Proceedings of the IEEE, vol. 87, no. 7, pp. 1079-1107, 1999.

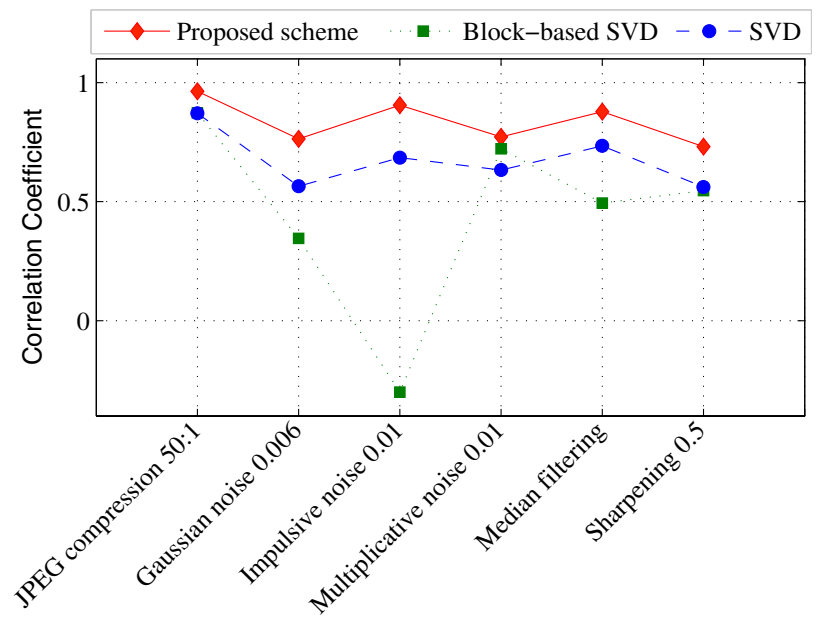

Fig. 6. Correlation coefficient comparison results between the proposed method and other schemes.

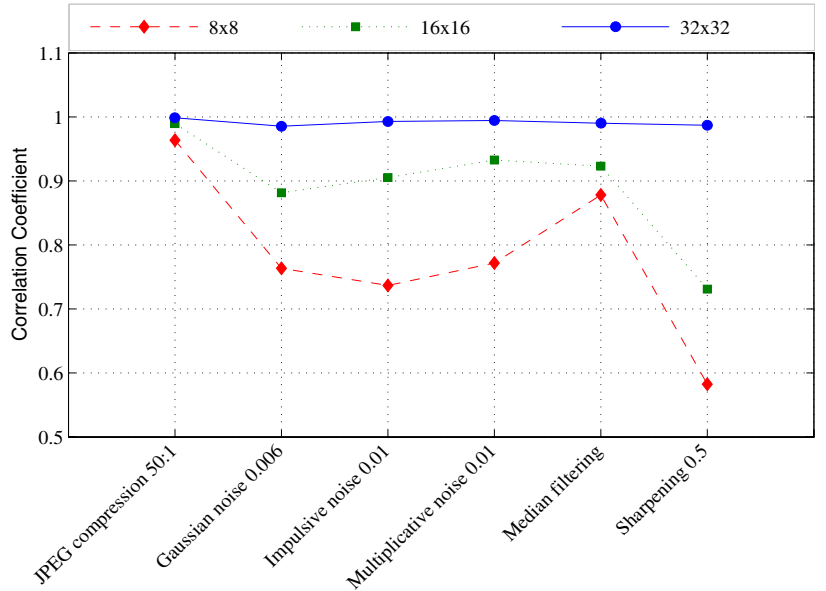

Fig. 7. Correlation coefficient results using three different block sizes.

[7] A. Sverdlov, S. Dexter, and A.M. Eskicioglu, "Robust DCT-SVD domain image watermarking for copyright protection: Embedding data in all frequencies," Proc. Euro. Sig. Proc. Conference, Turkey, 2005.

[8] R. Liu and T. Tan, "A SVD-based watermarking scheme for protecting rightful ownership," IEEE Trans. Multimedia, vol. 4, no. 1, pp. 121-128, 2002.

[9] E. Ganic, N. Zubair, and A.M. Eskicioglu, "An optimal watermarking scheme based on singular value decomposition," Proc. Communication, Network, and Information Security, 2003. 